\title{
A Proposal for a Genome Similarity-Based Taxonomy for Plant-Pathogenic Bacteria that Is Sufficiently Precise to Reflect Phylogeny, Host Range, and Outbreak Affiliation Applied to Pseudomonas syringae sensu lato as a Proof of Concept
}

\author{
Boris A. Vinatzer, Alexandra J. Weisberg, Caroline L. Monteil, Haitham A. Elmarakeby, Samuel K. Sheppard, \\ and Lenwood S. Heath
}

First, second, and third authors: Department of Plant Pathology, Physiology and Weed Science, Virginia Tech, Virginia; second author: Department of Botany and Plant Pathology, Oregon State University, Corvallis, Oregon; fourth and sixth authors: Department of Computer Science, Virginia Tech, Blacksburg, Virginia; and fifth author: Department of Biology \& Biotechnology, University of Bath, Claverton Down, Bath, United Kingdom.

Accepted for publication 12 August 2016.

\begin{abstract}
Taxonomy of plant pathogenic bacteria is challenging because pathogens of different crops often belong to the same named species but current taxonomy does not provide names for bacteria below the subspecies level. The introduction of the host range-based pathovar system in the 1980s provided a temporary solution to this problem but has many limitations. The affordability of genome sequencing now provides the opportunity for developing a new genome-based taxonomic framework. We already proposed to name individual bacterial isolates based on pairwise genome similarity. Here, we expand on this idea and

Using 93 genomes of Pseudomonas syringae sensu lato, LINs were compared with a $P$. syringae genome tree whereby the assigned LINs were found to be informative of a majority of phylogenetic relationships. LINs also reflected host range and outbreak association for strains of $P$. syringae pathovar actinidiae, a pathovar for which many genome sequences are available. We conclude that LINs could provide the basis for a new taxonomic framework to address the shortcomings of the current pathovar system and to complement the current taxonomic system of bacteria in general.
\end{abstract} propose to use genome similarity-based codes, which we now call life identification numbers (LINs), to describe and name bacterial taxa.
Additional keywords: average genome identity, core genome phylogeny.
Classification of bacteria has been a challenge for taxonomists since the beginning of microbiology. Because bacteria do not reproduce sexually, the biological species concept that describes species as groups of individuals that can breed together and produce fertile offspring (Mayr 1942) cannot be directly applied to asexually reproducing bacteria. Over the years, many alternative species concepts have thus been developed for bacteria (Mayden 1997). Since no consensus has yet been reached on which bacterial species concept is most appropriate, a pragmatic bacterial species concept is in use today, which defines bacterial species as monophyletic and genomically coherent clusters of strains characterized by a high degree of similarity in several independent characteristics (RosselloMora and Amann 2001). In practice, this system requires named bacterial species to be represented by a type strain that has a lower than 70\% DNA-DNA hybridization (DDH) value compared with the type strain of the most closely related named species. It has been shown that $70 \%$ DDH between the DNA of two bacteria corresponds approximately to a computationally determined $95 \%$ average nucleotide identity (ANI) calculated based on the genome sequences of the same two bacteria (Goris et al. 2007; Konstantinidis

Corresponding author: B. A. Vinatzer; E-mail address: vinatzer@vt.edu

First and second authors contributed equally to this work.

*The $\boldsymbol{e}$-Xtra logo stands for "electronic extra" and indicates that five supplementary tables and five supplementary figures are published online.

(C) 2017 The American Phytopathological Society and Tiedje 2005). Additionally, a set of phenotypic tests are required that differentiate a new species from related species (Rossello-Mora and Amann 2001).

The problem with the current taxonomic system is that it requires a lengthy and laborious process, from discovery of a new species to peer-reviewed publication of the description and the naming of the new species (Vinatzer and Bull 2009), a problem recently discussed in depth by Thompson and colleagues (Thompson et al. 2015). Similarly problematic is the fact that there is no general classification system to describe the extensive diversity that exists within many bacterial species, a problem we discussed in detail previously (Marakeby et al. 2014). The problem is particularly relevant to plant pathogenic bacterial species since several plant pathogenic species, such as Ralstonia solanacearum, Xanthomonas axonopodis, or Pseudomonas syringae, include pathogens of many different crops (Vinatzer and Bull 2009). This makes it impossible for plant pathologists to classify and name these pathogens using the current taxonomic system. It was attempted to temporarily address this problem by developing the pathovar system, which subdivides plant pathogenic species into so-called pathovars based on host range (Young et al. 1978). However, host range determination is not only laborious but also difficult to standardize and genetically distinct pathogens have sometimes very similar and overlapping host ranges, for example $P$. cannabina pv. alisalensis and $P$. syringae pv. maculicola (Bull et al. 2010; Cintas et al. 2002). Therefore, although the pathovar system is widely used today, few plant pathologists are satisfied with it.

Approaches such as multilocus sequence typing (MLST), which is based on the sequences of five to eight housekeeping genes 
(Maiden et al. 1998), or ribosomal MLST (rMLST), which is based on the sequences of 53 genes coding for ribosomal proteins (Jolley et al. 2012) have been widely used to type strains within animal and human pathogen species and, to some extent, plant pathogen species (Vinatzer et al. 2014). However, while MLST and rMLST are excellent tools to assign strains to named clonal lineages (called sequence types) or to named groups of closely related clonal lineages (called clonal complexes), these systems do not provide names for taxa at higher levels of genetic diversity.

We recently proposed to classify and name individual organisms, including individual bacterial and viral isolates, automatically using an exclusively genome similarity-based approach (Marakeby et al. 2014; Weisberg et al. 2015). We proposed that every individual organism (or virus) should be given a code that expresses how similar the organism is to other organisms. The proposed codes consist of a number of positions, with positions from the left to the right corresponding to increasing ANI thresholds. Therefore, two organisms that are very different from each other will not share any positions of their codes; two organisms with intermediate similarity will share the left-most positions of their codes; and, finally, almost identical organisms will share almost all positions of their codes (Fig. 1). In this system, ANI is not simply used to answer the question of whether two organisms belong-or do not belong-to the same species; rather ANI is translated into highly informative names that express how similar two organisms are. We now refer to these genome similarity-based codes as life identification numbers (LINs).

We already proposed that the LIN positions shared by all organisms belonging to a species could be included in species descriptions to indicate how genetically diverse a species is (Marakeby et al. 2014). Here we go one step further and use genome sequences of isolates of Pseudomonas syringae sensu lato to show that bacteria can be classified into named taxonomic groups independently of any species concept based solely on shared LIN positions. LIN-based taxa can either encompass what is currently considered a species complex, a species, an infraspecific group such as a pathovar, or even a single epidemic clone. $P$. syringae sensu lato is ideally suited to test the concept of LIN-based taxa, since it encompasses strains that, based on genome similarity, could be assigned to nine separate species (Gardan et al. 1999). However, the absence of consistent phenotypic differences between these nine groups has been a hindrance in describing these "genomo-species" as named species (Bull et al. 2011). Because of this, the main infraspecific classification for $P$. syringae sensu lato in use today is the above described pathovar system (Young et al. 1978). Therefore, a genome-based taxonomy that could describe bacterial diversity within named species (or even beyond named species) would be particularly useful for $P$. syringae sensu lato and other plantpathogenic bacteria but, as we will discuss, could be applied to other bacteria as well.

\section{MATERIALS AND METHODS}

All Pseudomonas genome sequences available at NCBI in April 2014, were downloaded. Genome sequences (Supplementary Table S1 provides a complete list) were imported into a BIGSdb (Jolley and Maiden 2010) database maintained at the Institute of Life Science, College of Medicine, Swansea University (Swansea, United Kingdom).

The sequences corresponding to the $53 \mathrm{rps}$ genes used in rMLST (Jolley et al. 2012) were identified in 486 genome sequences by tagging the reference type sequences of $53 \mathrm{rps}$ genes retrieved by Jolley et al. (2012) using blastn (Altschul et al. 1997) with a cut-off of $70 \%$ identity over $50 \%$ of the alignment length and a word size of 15. Tagged sequences were then aligned using MAFFT (Katoh and Standley 2013) and concatenated. A maximum likelihood (ML) phylogenetic tree was constructed using RAxML (Stamatakis 2014) with $50 \mathrm{ML}$ search replicates and 1000 nonparametric bootstrap replicates under the GTRGAMMA model. Nonparametric bootstrap branch support values were mapped as a percentage onto the best log-likelihood ML tree, and clades with less than $50 \%$ bootstrap support were collapsed into polytomies using TreeCollapseCL 4 (Hodcroft 2015).

Core genome genes (genes shared by at least $90 \%$ of the represented genomes) were identified separately for the four main clades of the rMLST tree, and core genome gene alignments were generated using the Genome comparator function of BIGSdb (Jolley and Maiden 2010). Core genome trees were built for all genomes consisting of fewer than 1,000 contigs using the same approach that was used for the rMLST tree, with the exception of the use of the GTRCAT model for the $P$. syringae, $P$. aeruginosa, and $P$. fluorescens datasets. This model was selected for the larger datasets in order for the analysis to run to completion using the memory available on the compute cluster. Branch lengths and model parameters of each final tree were then optimized under the GAMMA model.

LINs were assigned as previously described (Marakeby et al. 2014) to all genome sequences included in the rMLST tree using the LIN position thresholds shown in Figure 1. Taxonomic groupings indicated by shared LIN positions between taxa in the $P$. syringae sensu lato dataset were represented as a cladogram by building a prefix tree of LINs whereby bi- and multifurcations were assigned where LIN positions differed (going from the left to the right of the LINs). The branch structure of the LIN-based cladogram and the ML tree were then compared by creating a consensus network using SplitsTree4 (Huson and Bryant 2006).

\section{RESULTS}

Clades of a phylogenetic tree of the genus Pseudomonas based on rMLST correspond to the main Pseudomonas species but have low statistical support for infraspecific groupings. To obtain a first idea of the phylogenetic relationships among the 487 Pseudomonas strains for which genome assemblies were available

\begin{tabular}{|c|c|c|}
\hline $\begin{array}{l}\% \text { identity cutoff } \\
\text { Position }\end{array}$ & 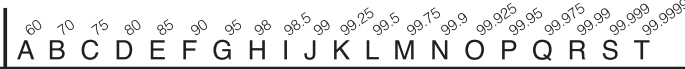 & $\begin{array}{l}\% \text { ANI to most } \\
\text { similar genome }\end{array}$ \\
\hline Pav-BPIC_631 & 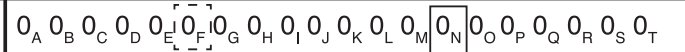 & 1003 \\
\hline Pav-CRAFRUec1 & $0_{A} 0_{B} 0_{C} 0_{D} 0_{E} 0_{F} 0_{G} 0_{H} 0,0_{J} 0_{K} 0_{L} 0_{M}\left|1_{N}\right| 0_{O} 0_{P} 0_{Q} 0_{R} 0_{S} 0_{T}$ & 99.76 \\
\hline Psa-NCPPB_3335 & 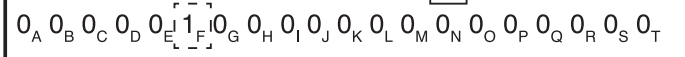 & 87.28 \\
\hline$\vdots$ & $\vdots$ & \\
\hline Psy-CC440 & 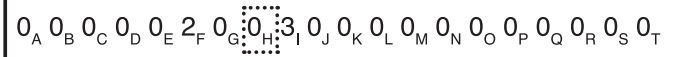 & 98.19 \\
\hline$\vdots$ & 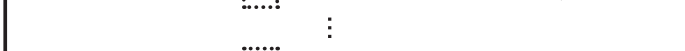 & \\
\hline Psy-DSM10604 & 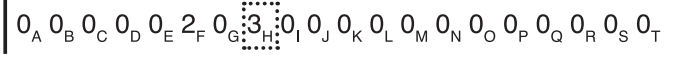 & 97.99 \\
\hline
\end{tabular}

Fig. 1. Thresholds corresponding to life identification number (LIN) positions and examples of LIN assignment based on the average nucleotide identity (ANI) between the genome sequence of a new strain and the most similar genome of a strain that has already an assigned LIN. The LIN position at which a strain differs compared with its most similar strain is indicated with a box. 
in GenBank in April 2014, the 53 genes coding for ribosomal proteins were extracted from each genome sequence to build a single rMLST (Jolley et al. 2012) tree (Fig. 2A). Francisella TX077308 was used as outgroup. Most branches in the tree, particularly those separating different species, had strong bootstrap support. However, the tree also revealed the limitation of rMLST: many smaller clades (particularly within-species clades) had low bootstrap values of less than $90 \%$, and many clades had bootstrap support of less than 50\% and were collapsed into polytomies (see Figure 2B for such an example in a $P$. syringae sensu lato subclade). Therefore, rMLST was not sufficient for obtaining a deep phylogeny of $P$. syringae sensu lato and of other groups within the genus Pseudomonas.
A core genome tree of $P$. syringae sensu lato has strong statistical support for infraspecific groupings. All genomes of $P$. syringae sensu lato strains clustered together in the rMLST tree with $100 \%$ bootstrap support (Fig. 2). For this group, the core genome, i.e., all genes shared by $90 \%$ of genomes of this group, was determined. The identified 2,726 core genome genes were then aligned, and an ML tree was built. A cladogram is shown in Figure 3, and a tree with proportional branch length is shown in Supplementary Figure S1. Compared with the P. syringae sensu lato clade of the rMLST tree, this core genome tree has many more clades with bootstrap values of over 90\%: 76 compared with 36.

A

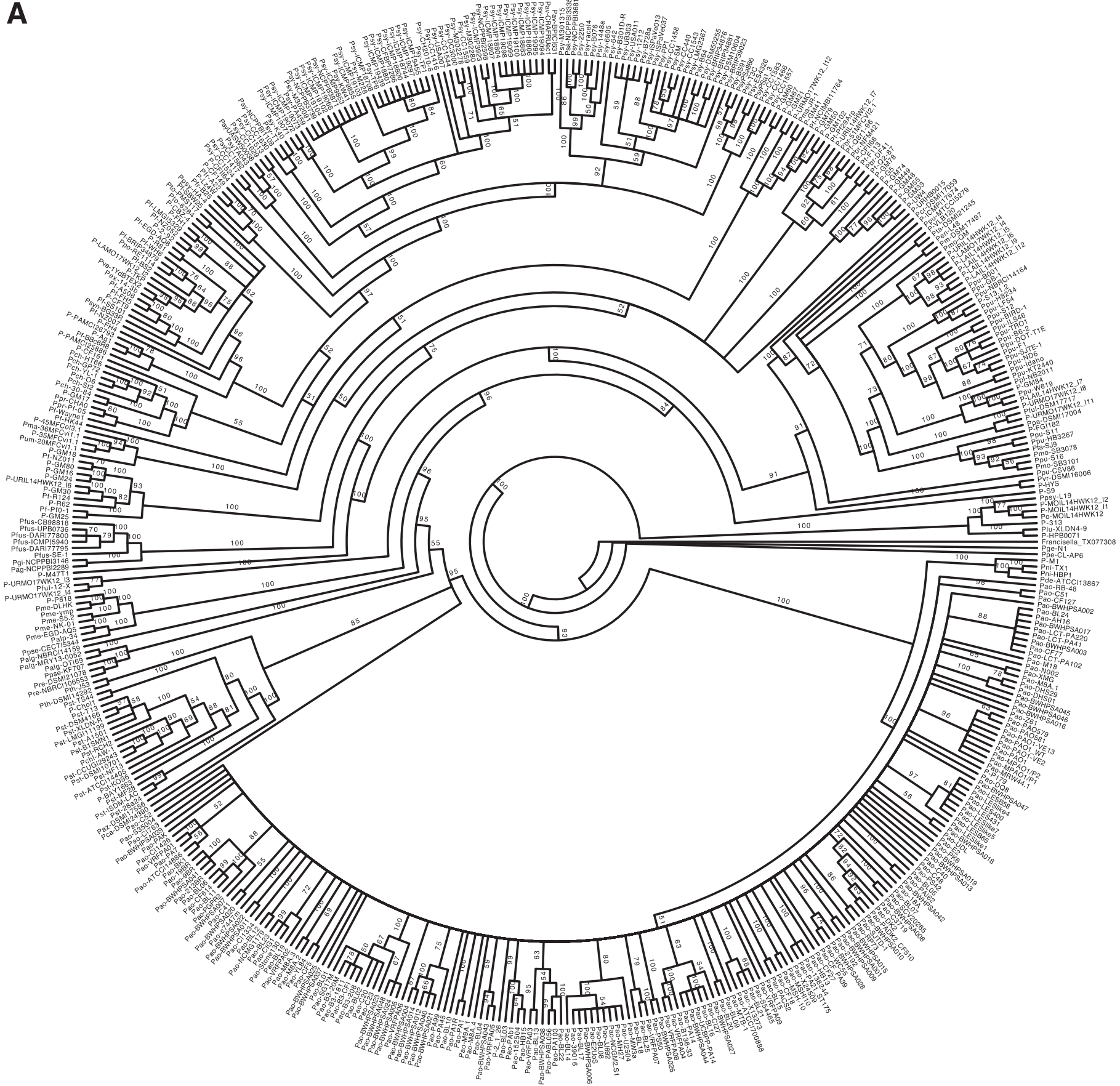

Continued on next page

Fig. 2. Phylogenetic tree based on the alignment of 53 ribosomal multilocus sequence typing (rMLST) loci. A, Unrooted maximum likelihood tree. B, A section of the tree including Pseudomonas syringae pv. actinidiae strains showing that rMLST does not provide a deep phylogeny. Branch support values are displayed as a percentage of 1,000 nonparametric bootstrap replicates. Branches with less than 50\% bootstrap support were collapsed into polytomies. Branch lengths are not to scale. Pao: P. aeruginosa, Pf: P. fluorescens, Ppu: P. putida, Pst: P. stutzeri, Psy: P. syringae. 
Separate core genome trees (Supplementary Figs. S2, S3, and S4) were built for three other clades of the rMLST tree, roughly corresponding to $P$. fluorescens plus $P$. stutzeri (555 core genome genes), $P$. aeruginosa (2,134 core genome genes), and $P$. putida including related species (137 core genome genes; the number of

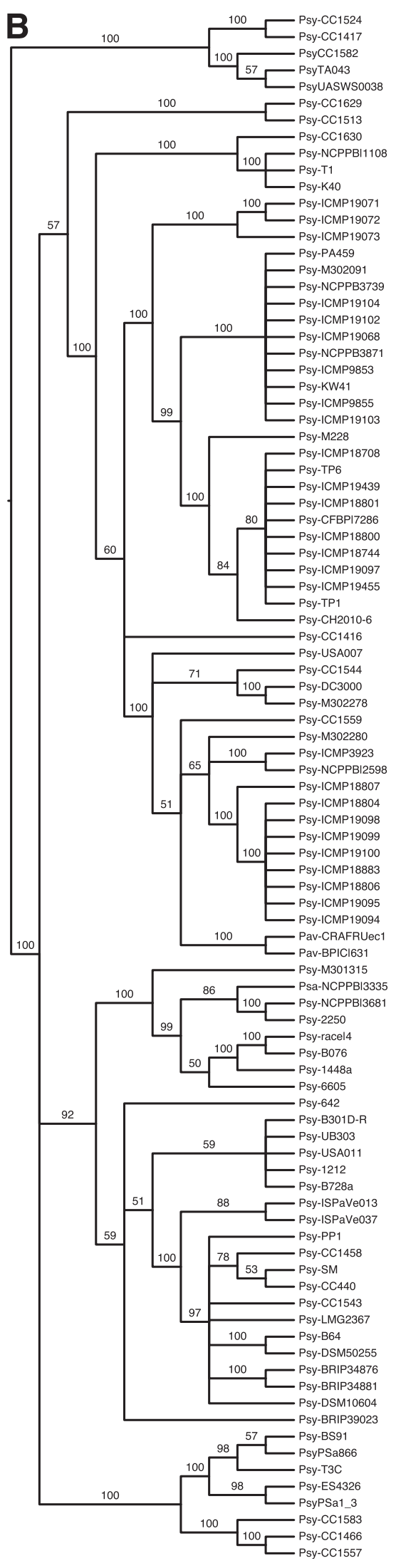

Fig. 2. Continued from previous page. core genome genes in this groups is much smaller compared with the other groups because this group of strains is genetically much more diverse). The three trees confirmed the higher discriminatory power of core genome phylogenies compared with rMLST.

Unique LINs were assigned to each $P$. syringae sensu lato genome. After constructing the phylogenetic trees based on core genome genes, we assigned LINs to every $P$. syringae sensu lato strain using our previously described pipeline, which uses jSpecies (Richter and Rosselló-Móra 2009) to calculate pairwise ANI values based on all genomic regions that align between the two genomes that are being compared. The number of LIN positions that were used was reduced from 24 to 20 compared with our two previous publications (Marakeby et al. 2014; Weisberg et al. 2015); several LIN positions with high ANI values (99.91, 99.92, 99.93, 99.94, 99.6, 99.7, and 99.8) were eliminated and replaced by one new position with an ANI values of 99.25 because very few Pseudomonas genomes had ANI values in this range. On the other hand, two additional LIN positions with relatively low ANI values were added $(75,98.5)$ because the ANI between several genomes was in this lower range of values. In other words, the set of genomes that were compared here was more divergent overall compared with genomes analyzed in our previous two publications, and therefore ANI thresholds were slightly adjusted. LIN thresholds are shown in Figure 1. Note that the LIN positions and thresholds used here and the LINs assigned here are not intended to be permanent. LINs were assigned here to show the potential of the LIN system. Permanent LIN positions and thresholds will only be chosen and permanent LINs will only be assigned after a permanent LIN database has been established (see Discussion section).

A short summary of LIN assignment follows to clarify the procedure (Fig. 1). A LIN consisting of all zeroes (instead of zeroes any other number or letter or symbol could have been used) was assigned to one genome, in our case, the genome of the strain PavBPIC_631, since it was the first genome in alphabetical order in our list of $P$. syringae sensu lato genomes. Then, the ANI between the second genome in our list, Pav-CRAFRUec1, and Pav-BPIC_631 was determined. The ANI value between the two genomes was determined to be $99.7631 \%$. Therefore, Pav-CRAFRUec1 was assigned an identical LIN to strain BPIC_631 up to position M corresponding to the $99.75 \%$ threshold, because the two strains are over $99.75 \%$ identical to each other. However, strain CRAFRUec1 was assigned a " 1 " at position N (solid box in Fig. 1) corresponding to the $99.9 \%$ threshold because the two strains are less than $99.9 \%$ identical (instead of a "1" any other number, letter, or symbol could have been assigned; it only matters that it is not a " 0 "). Therefore, the difference between the LINs of the two strains at this position reflects the level of genomic difference between these two strains. The genome of the third strain in our table, the strain labeled Psa-NCPPB_3335, was then compared with the genomes of the first two strains. Strain NCPPB_3335 was found to me more similar to strain BPIC_631 than to strain CRAFRUec 1 with an ANI value of $87.28 \%$. Therefore, a LIN was assigned to strain NCPPB_3335 based on strain CRAFRUec1. The LIN of strain NCPPB_3335 is identical up to position E corresponding to the $85 \%$ threshold, because the two strains have an ANI higher than this threshold but they are different at position $\mathrm{F}$ because their ANI is lower than the threshold at position F, i.e., $90 \%$ (dashed boxes in Fig. 1). This was continued until a LIN was assigned to the last strain in our list of $P$. syringae sensu lato genomes, the strain labeled as Psy-DSM10604, whose genome was most similar to the genome of strain CC440 (ANI value of 97.99\%). Strain DSM10604 was thus assigned a LIN based on strain CC440 and the two strains share the same LIN up to position G corresponding to the $95 \%$ threshold but are different at position $\mathrm{H}$ corresponding to the $98 \%$ threshold (dotted boxes in Fig. 1). LINs were mapped to each strain in Figure 3. Moreover, Supplementary Table S2 lists, for each strain, the genome based on which the LIN was assigned, the ANI compared with that genome, and the LIN.

The LIN pipeline was run separately for $P$. fluorescens plus $P$. stutzeri, $P$. putida, and $P$. aeruginosa, and LINs were mapped to 
the strains in the respective trees. Supplementary Tables S3, S4, and S5 show, for each strain, the genome based on which the LIN was assigned, the ANI compared with that genome, and the LIN.
LINs and core genome phylogeny are in agreement. Although LINs could be useful for simply cataloging and consistently naming individual strains, even if they were not predictive of phylogenetic relationships (as discussed in detail previously

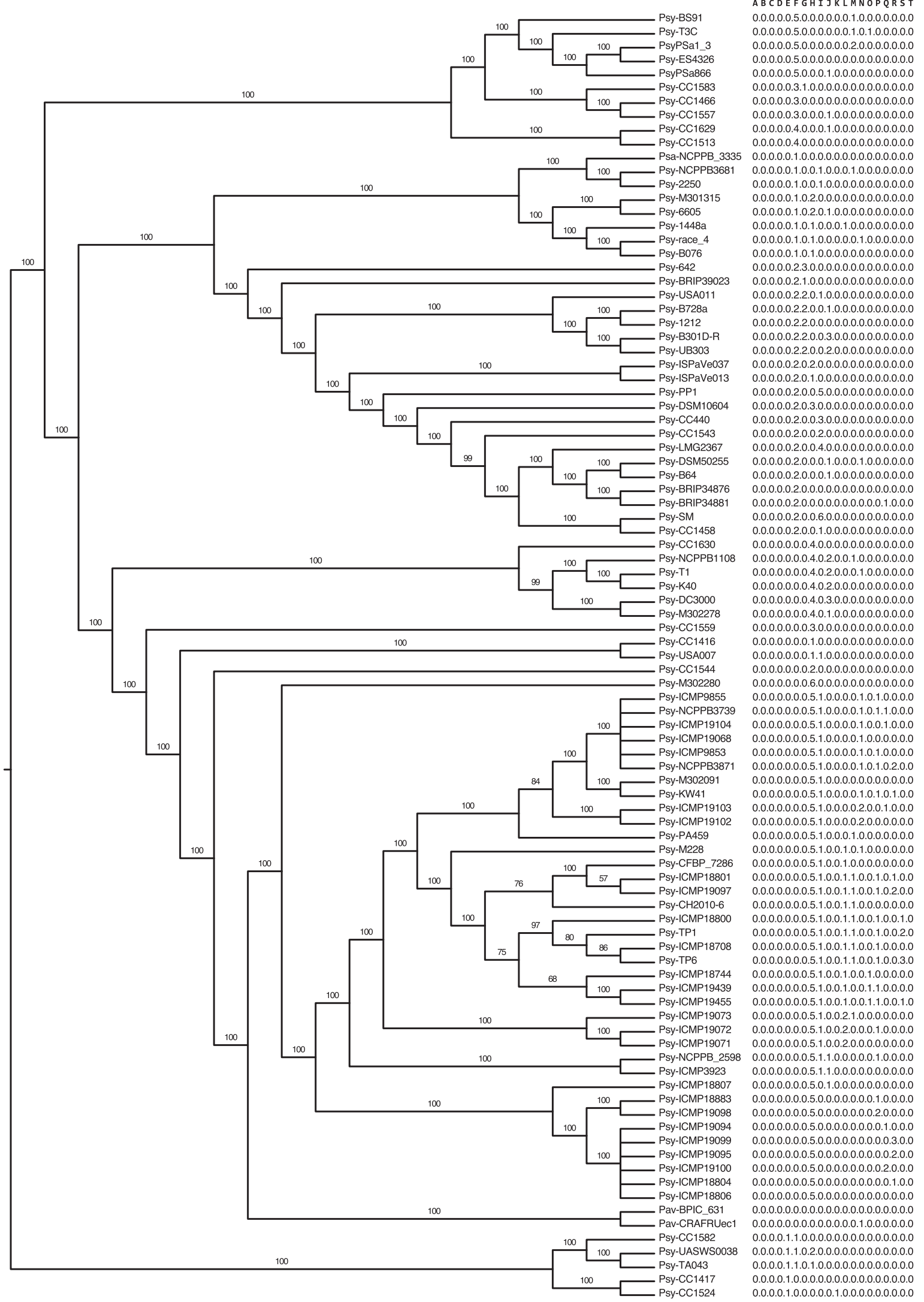

Fig. 3. Pseudomonas syringae sensu lato core genome maximum likelihood tree (cladogram) with life identification numbers (LINs) mapped to each strain. LIN positions are labeled at the top of the figure. Branch support values are displayed as a percentage of 1,000 nonparametric bootstrap replicates. Branches with less than $50 \%$ bootstrap support were collapsed into polytomies. Branch lengths are not to scale. 
(Marakeby et al. 2014)), LINs need, of course, to be in agreement with phylogenetic relationships if we want to use them as a phylogenetic framework for taxonomy. Therefore, the core genome trees of $P$. syringae sensu lato and of the other Pseudomonas groups were now compared with the assigned LINs. This was done by counting the occurrences of the following three situations for all clades with bootstrap support of over 90 (Table 1): (i) clades that were uniquely identified by a single LIN position (for example, the number 1 at position $F\left[1_{F}\right]$ clearly identifies the clade labeled "II" in Figure 4); (ii) clades that can be described by a group of numbers at a certain LIN position (for example, the numbers 3,4 , and 5 at position $\mathrm{F}\left[3,4,5_{\mathrm{F}}\right]$ are exclusively associated with strains present in the clade labeled "I" in Figure 4); and (iii) clades that cannot be described by a single LIN position because there is not a single LIN position that can distinguish members of a clade from members outside of the clade (for example, in Figure 4, Psy-T3C is a member of clade "A" and shares a 1 at position M with Psy-BS91 outside of clade "A" but not with PsyPSa1_3, Psy-ES4326, and PsyPSa866 [which are the other members of clade "A"]). However, note that even in this last situation, clades can still be described by the LINs of the member strains, but more than one LIN position needs to be used in the description (for example, in Figure 4, clade " $\mathrm{A}$ " can be described as $0_{\mathrm{J}} 0_{\mathrm{K}} 0_{\mathrm{L}} 1_{\mathrm{M}} 0_{\mathrm{N}} 1_{\mathrm{O}}, 0_{\mathrm{J}} 0_{\mathrm{K}} 0_{\mathrm{L}} 2_{\mathrm{M}} 0_{\mathrm{N}} 0_{\mathrm{O}}, 0_{\mathrm{J}} 0_{\mathrm{K}} 0_{\mathrm{L}} 0_{\mathrm{M}} 0_{\mathrm{N}} 0_{\mathrm{O}}$, $\left.1_{\mathrm{J}} 0_{\mathrm{K}} 0_{\mathrm{L}} 0_{\mathrm{M}} 0_{\mathrm{N}} 0_{\mathrm{O}}\right)$.

In case 1 , LINs and phylogeny agree and the same information present in the tree is reflected in the LINs. In case 2, LINs do not

TABLE 1. Correlations between life identification numbers (LINs) and clades of core genome phylogenies (only clades with a bootstrap support of over 90 were considered)

\begin{tabular}{lccc}
\hline & $\begin{array}{c}\text { Number of } \\
\text { clades that can } \\
\text { be described by a } \\
\text { single number at } \\
\text { a single LIN } \\
\text { position }\end{array}$ & $\begin{array}{c}\text { Number of clades } \\
\text { that can be } \\
\text { described by more } \\
\text { than one number } \\
\text { at a single LIN } \\
\text { position }\end{array}$ & $\begin{array}{c}\text { Number of } \\
\text { clades that need } \\
\text { to be described } \\
\text { by more than a } \\
\text { single LIN } \\
\text { position }\end{array}$ \\
\hline $\begin{array}{l}\text { Pseudomonas } \\
\text { syringae }\end{array}$ & 41 & & \\
$\begin{array}{l}\text {. fluorescens } \\
\text { and P. stutzeri }\end{array}$ & 54 & 27 & 8 \\
$\begin{array}{l}P \text { p putida } \\
\text { P. aeruginosa }\end{array}$ & 30 & 53 & 11 \\
\hline
\end{tabular}

a One or more strains inside a clade shared the same number at an LIN position with one or more strains in the clade immediately basal to it. contradict phylogeny, but some phylogenetic relationships present in the tree are not reflected in the LINs. In all occurrences of case 3, one or more strains in a clade shared a LIN position with the strain immediately basal to the clade. In other words, in these occurrences LINs moved strains out of a clade into the next larger clade. We did not find a single situation where a strain within a clade shared a LIN position with a strain beyond the branch immediately basal to the clade. Therefore, LINs in no case contradicted core genome phylogeny besides changing the branching order by one position.

Finally, LINs themselves were used to build a cladogram of P. syringae sensu lato (Fig. 5), and this cladogram was compared with the corresponding core genome cladogram by building a consensus network out of the two cladograms (Fig. 6). If the two cladograms contradicted each other, a certain number of reticulations would be expected. However, there is not a single visible reticulation in the consensus network, confirming that LINs are in very good agreement with core genome phylogeny. Moreover, as a control, we switched two strain names in the core genome tree and then rebuilt the consensus network. Reticulations can now easily be seen confirming that the network would have revealed incongruences between LINs and core genome phylogeny if they had been present (Supplementary Fig. S5).

LINs are predictive of pathogenicity and disease outbreaks; the example of bacterial canker of kiwifruit. Bacterial canker of kiwifruit is an emerging disease caused by different, but closely related, epidemic clones of a group of $P$. syringae strains described as $P$. syringae pv. actinidiae based on their pathogenicity on kiwifruit (Actinidia deliciosa, A. sinensis, and other related species) (Butler et al. 2013; Mazzaglia et al. 2012; McCann et al. 2013; Takikawa et al. 1989). Within P. syringae pv. actinidiae, plant pathologists have distinguished different subgroups based on virulence (Chapman et al. 2011), geographic origin (Marcelletti et al. 2011; Mazzaglia et al. 2011), and genetics/genomics (Chapman et al. 2012). More recently, some strains from New Zealand and Australia, which were isolated from kiwifruit plants but only cause leaf spots instead of cankers, were described as pv. actinidifoliorum (Cunty et al. 2014). This situation is very confusing. In the following paragraphs we show how LINs could alleviate this confusion and give new insights not only into phylogeny but pathogenicity and epidemiology as well.

In Figure 7, we zoom in on the section of the $P$. syringae sensu lato core genome tree that contains $P$. syringae pv. actinidiae. Labels were added to various clades based on the literature cited above. Comparing Figure 3 and Figure 7 and looking at the LINs from the

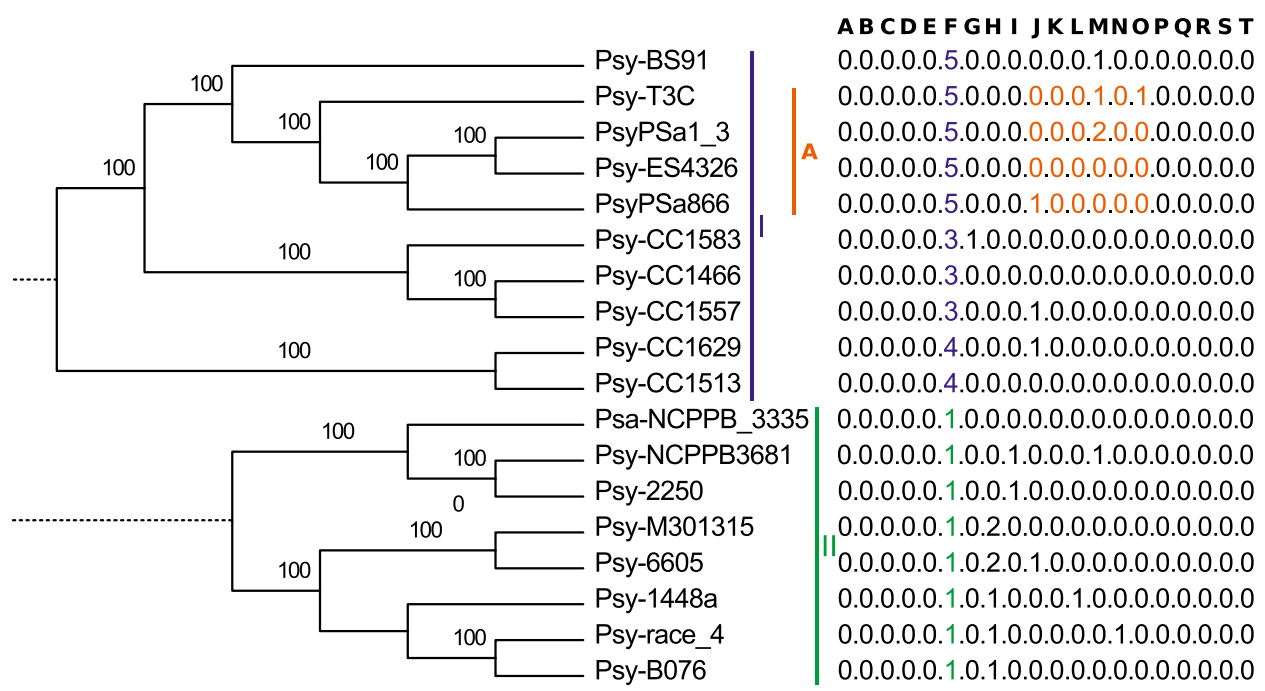

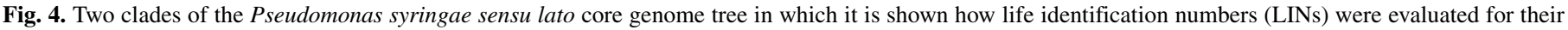
representation of phylogenetic relationships. LIN positions are labeled at the top of the figure. 
left to the right, it can be seen how LIN positions from A to D are shared by $P$. syringae pv. actinidiae with all $P$. syringae sensu lato strains (Fig. 3). Therefore, $0_{\mathrm{A}} 0_{\mathrm{B}} 0_{\mathrm{C}} 0_{\mathrm{D}}$ defines $P$. syringae as a species complex and $P$. syringae pv. actinidiae as a member of this complex.

LIN position $\mathrm{E}$ is shared by $P$. syringae pv. actinidiae with most $P$. syringae sensu lato strains $\left(0_{\mathrm{E}}\right)$, besides a small group of environmental strains that are located in a separate clade in the $P$. syringae sensu lato tree (at the very bottom) and that are distinguished from all other $P$. syringae strains by LIN $1_{\mathrm{E}}$.

LIN positions $\mathrm{F}$ and $\mathrm{G}$ are shared by $P$. syringae pv. actinidiae strains with several other $P$. syringae sensu lato strains $\left(0_{\mathrm{F}} 0_{\mathrm{G}}\right)$, for example, with the $P$. syringae pv. tomato strains Psy-NCPPB1108,
Psy-T1, Psy-K4, and Psy-DC3000. Therefore, the shared LIN positions $0_{\mathrm{F}} 0_{\mathrm{G}}$ indicate that $P$. syringae pv. actinidiae is more closely related to the tomato-pathogenic $P$. syringae strains than, for example, to the $P$. syringae strains at the top of Figure 3, which were discussed earlier and that have the LINs $3_{\mathrm{F}}, 4_{\mathrm{F}}$, and $5_{\mathrm{F}}$.

LIN position $\mathrm{H}$ indicates that $P$. syringae pv. actinidiae and $P$. syringae pv. actinidifoliorum are closely related to the teapathogenic strains of pathovar theae, since all three share a 5 in position $\mathrm{H}\left(5_{\mathrm{H}}\right)$. However, $P$. syringae pv. actinidifoliorum is separated from $P$. syringae pv. actinidiae and from $P$. syringae $\mathrm{pv}$. theae strains by LIN position I at which $P$. syringae pv. actinidiae and $P$. syringae pv. theae share a $1\left(1_{\mathrm{I}}\right)$, while $P$. syringae pv. actinidifoliorum strains have a $0\left(0_{\mathrm{I}}\right)$. LIN position I thus informs us

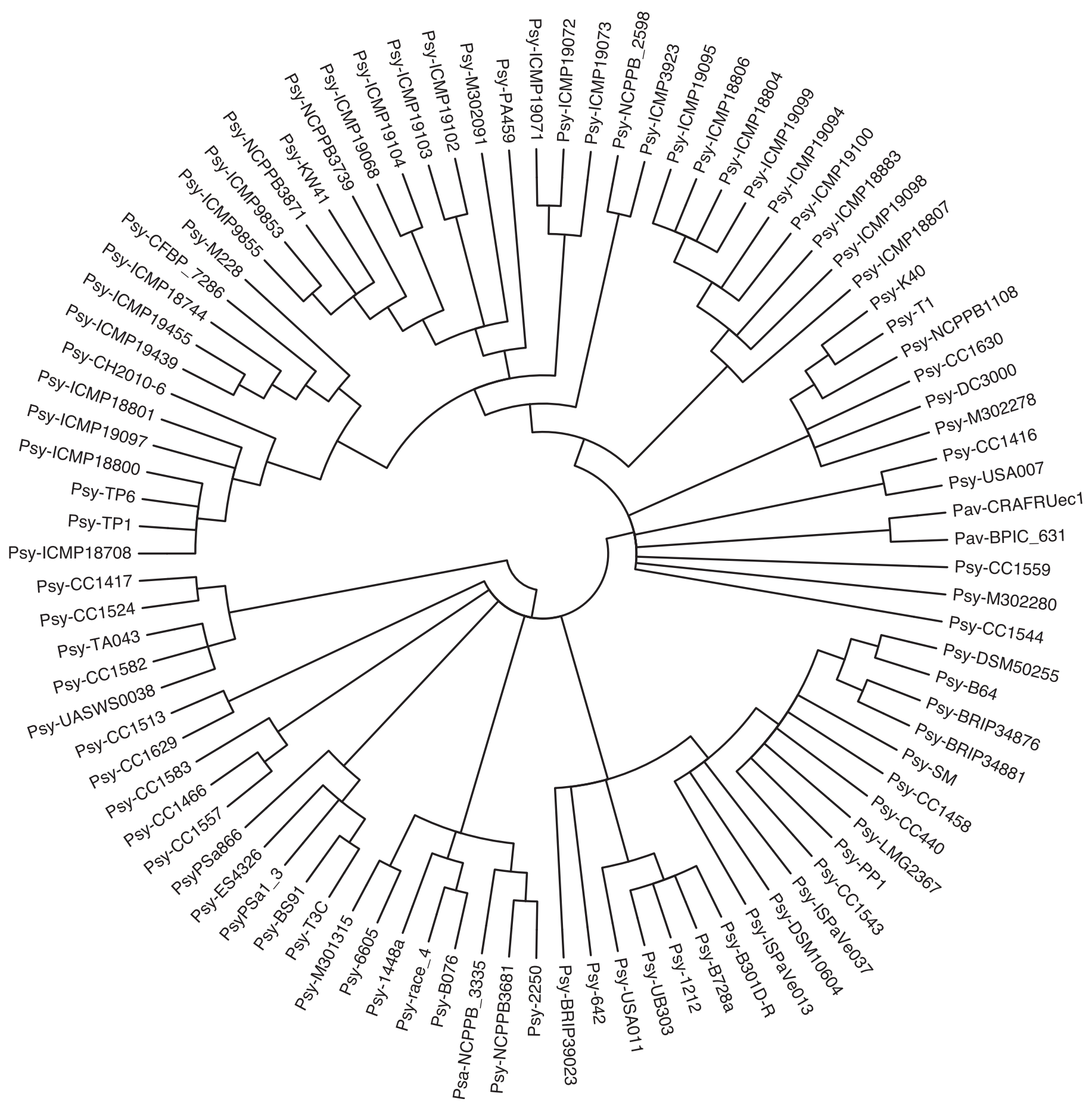

Fig. 5. Cladogram representation of life identification numbers (LINs) assigned to Pseudomonas syringae sensu lato genomes. Shared values or differences at each LIN position going from left to right (increasing LIN thresholds) are represented as splits in the tree. 
of the closer relationship between $P$. syringae pv. actinidiae and pathovar theae compared with pathovar actinidifoliorum.

LIN positions $\mathrm{J}$ and $\mathrm{K}$ finally uniquely identify $P$. syringae $\mathrm{pv}$. actinidiae strains, since strains isolated from kiwifruit that cause canker all share the same LIN from position A to position $\mathrm{K}$ : $0_{\mathrm{A}} 0_{\mathrm{B}} 0_{\mathrm{C}} 0_{\mathrm{D}} 0_{\mathrm{E}} 0_{\mathrm{F}} 0_{\mathrm{G}} 5_{\mathrm{H}} 1_{\mathrm{I}} 0_{\mathrm{J}} 0_{\mathrm{K}}$. Therefore, this LIN does not only precisely define $P$. syringae pv. actinidiae phylogenetically, but it is also predictive of $P$. syringae pv. actinidiae strains' ability to cause canker on kiwifruit, i.e., their pathogenicity.

Within P. syringae pv. actinidiae, all Japanese strains and one single Italian strain isolated from the same epidemic in the 1980s (Scortichini 1994; Takikawa et al. 1989) share LIN 0L, while all strains isolated during the more recent epidemic, which probably started in China and then spread to Europe, New Zealand, and Chile (Butler et al. 2013; Mazzaglia et al. 2012), are uniquely identified by LIN $1_{L}$. A small group of Korean strains, which are not epidemiologically connected to either epidemic (Chapman et al. 2012; Mazzaglia et al. 2012), have the LIN $2_{L}$. Therefore, LIN position $L$ is clearly predictive of the two main epidemics of canker of kiwifruit. Even within the more recent epidemic caused by the $1_{\mathrm{L}}$ strains, LINs appear to be able to distinguish some transmission chains from each other. For example, the two Chilean isolates, which are probably derived from a single introduction into this country, are uniquely identified by the LIN $0_{\mathrm{M}} 0_{\mathrm{N}} 1_{\mathrm{O}} 1_{\mathrm{P}}$.

Therefore, the $P$. syringae pv. actinidiae example shows that LINs can provide a very deep taxonomy, which is predictive of phylogeny and host range, as well as epidemiology.

\section{DISCUSSION}

Current bacterial classification makes a clear distinction between taxonomy (identification, classification, and naming of bacteria to the genus, species, and subspecies level) and strain typing (identification of phylogenetic relationships among strains that belong to the same species or subspecies). However, in the face of an emerging disease epidemic, such as canker of kiwifruit, the question of whether the pathogen that causes the disease should be considered a new named species or a new strain that belongs to an existing species becomes secondary. What is needed instead is a way to precisely identify and classify the pathogen and to give it a precise and informative name that can be used to communicate about this pathogen. While next generation sequencing now allows us to precisely and rapidly identify any new pathogen strain, unfortunately, current taxonomy does not allow us to precisely and rapidly name new pathogen strains (as eloquently described in (Thompson et al. 2015)). Here we have shown how a purely genome similarity-based species-independent taxonomy can do exactly that for $P$. syringae sensu lato and other pseudomonads.

First, we have shown here that the 53 genes used in rMLST are insufficient to precisely type Pseudomonas strains; many strains are assigned to phylogenetic clades with poor statistical support. Moreover, the basic units of MLST/rMLST, i.e., sequence types and clonal complexes, provide only limited information about phylogeny. Membership in the same clonal complex informs about the close relationships between sequence types that belong to that complex. However, relationships between clonal complexes or between sequence types that are not part of the same clonal complex are not provided by MLST. This does not mean that MLST/rMLST trees do not contain this information: the trees contain this information but this information is not reflected in the names that are being assigned to sequence types and clonal complexes.

The core genome tree constructed here for P. syringae sensu lato provided a much deeper and statistically better-supported

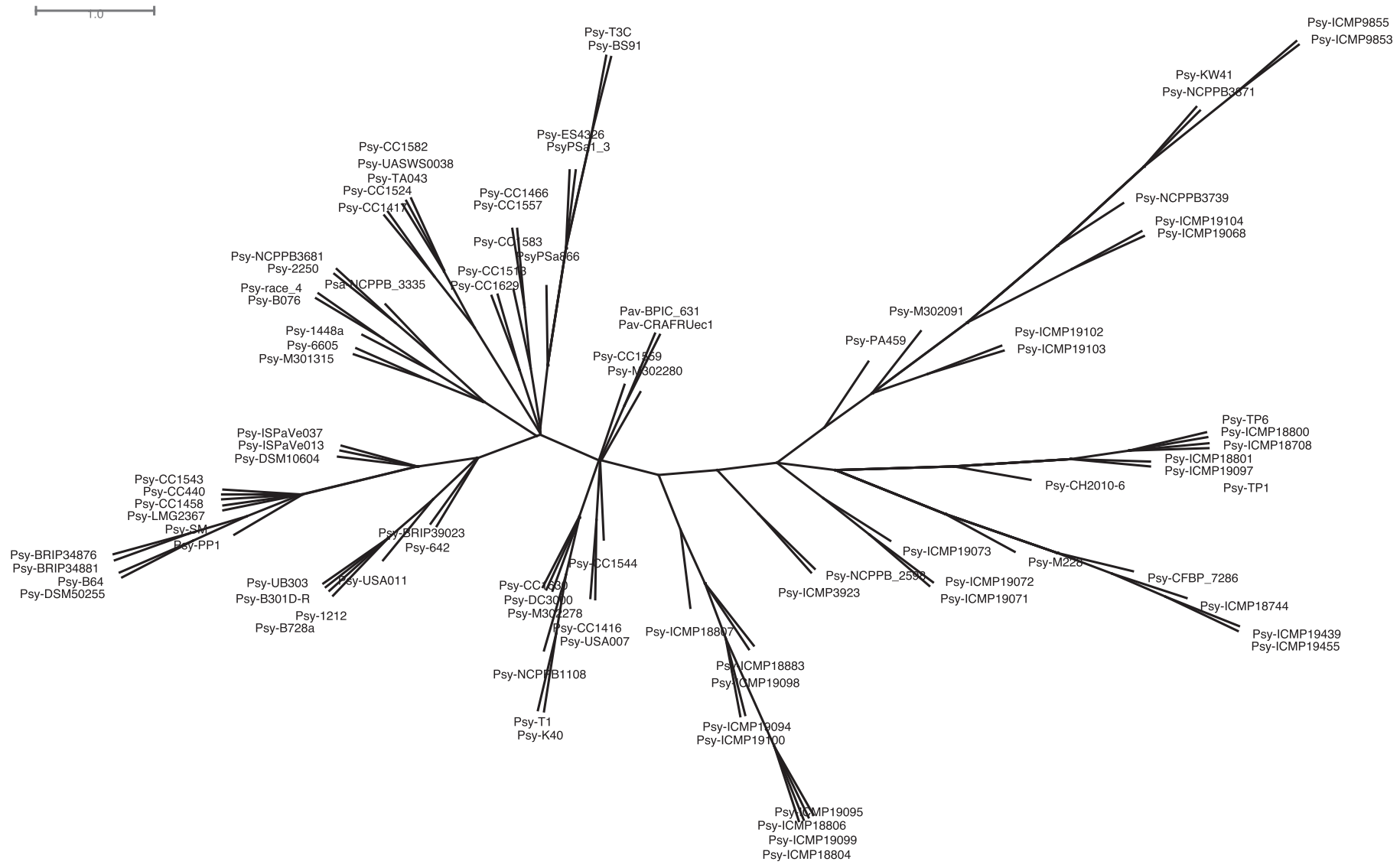

Fig. 6. Consensus Network built from the Pseudomonas syringae sensu lato core genome tree (Fig. 3) and the life identification number (LIN) tree (Fig. 5). Differences in topology between the core genome tree and the LIN-based cladogram are represented as reticulations in the network. Branch lengths are not to scale. 
phylogeny compared with rMLST. Could such a tree be used directly as the basis for a deep genome-based taxonomy? Yes, one could develop a hierarchical naming system that is based on clade and subclade membership. This system would precisely reflect phylogeny. In fact, clades in a phylogenetic tree (such as the tree in Fig. 3) could be labeled with letters or numbers to create a phylogenybased infra-specific classification, as was done for P. syringae in the past (Berge et al. 2014; Sarkar and Guttman 2004). However, the addition of new clades and the subdivision of existing clades into subclades upon discovery of new diversity would make such naming schemes unstable and confusing.

This brings us to the main advantage of LINs compared with taxonomy based on phylogenetic reconstruction: LINs are assigned sequentially to individual strains as their genome sequences become available, and previously assigned LINs do not change upon assignment of LINs to newly discovered strains. Therefore, the LINs assigned to individual strains provide a stable reference system for taxonomy and will not need to change when new taxa are described. This advantage of LINs was described in detail in a recent opinion paper (Baltrus 2016). However, do LINs nonetheless reflect phylogeny? This question can be divided into three more specific questions that are all three at least partially answered in our results: (i) Are LINs informative of all phylogenetic information that can be derived from whole genomes? (ii) Is the information encoded in LINs in agreement with the phylogeny inferred from whole genomes? (iii) Is phylogeny inferred from whole genomes even representative of evolutionary relationships?

As for the first question, the answer is no. We have shown here, for example, that 35 of the 76 clades in the P. syringae sensu lato core genome tree that have a bootstrap support of over 90 are not represented by a single number/symbol at a single LIN position. There are two reasons for that, which can be best explained using examples in the clade labeled "I" in Figure 4, which consists of three subclades identified by LIN position F: $3_{\mathrm{F}}, 4_{\mathrm{F}}$, and $5_{\mathrm{F}}$.

Example 1: Psy-ES4326 and Psy-CC1583 have an ANI of 89.325 and Psy-ES4326 was assigned LIN $0_{\mathrm{A}} 0_{\mathrm{B}} 0_{\mathrm{C}} 0_{\mathrm{D}} 0_{\mathrm{E}} 5_{\mathrm{F}}$ because it is over $85 \%$ identical, but less than $90 \%$ identical, to Psy-CC1583, which has LIN $0_{\mathrm{A}} 0_{\mathrm{B}} 0_{\mathrm{C}} 0_{\mathrm{D}} 0_{\mathrm{E}} 4_{\mathrm{F}}$. However, if instead of choosing a $90 \%$ threshold for position $\mathrm{F}$, we had chosen an $87.5 \%$ threshold, then Psy-ES4326 and Psy-CC1583 would share the same LIN at position $\mathrm{F}\left(4_{\mathrm{F}}\right)$ revealing that they both belong to clade I. Therefore, finetuning thresholds and possibly including additional LIN positions would improve representation of clades in LINs.

Example 2: Psy-CC1513 $\left(0_{\mathrm{A}} 0_{\mathrm{B}} 0_{\mathrm{C}} 0_{\mathrm{D}} 0_{\mathrm{E}} 4_{\mathrm{F}}\right)$ is more similar to Pav-CRAFRUec $1\left(85.88 \%\right.$ ANI) with LIN $0_{A} 0_{B} 0_{C} 0_{D} 0_{E} 0_{F}$ than to Psy-CC1466 (85.52\% ANI) with LIN $0_{\mathrm{A}} 0_{\mathrm{B}} 0_{\mathrm{C}} 0_{\mathrm{D}} 0_{\mathrm{E}} 3_{\mathrm{F}}$. Therefore, changing the threshold for position $\mathrm{F}$ to $87.5 \%$ would still not group Psy-CC1513 together with Psy-CC1466 and with Psy-ES4326 and

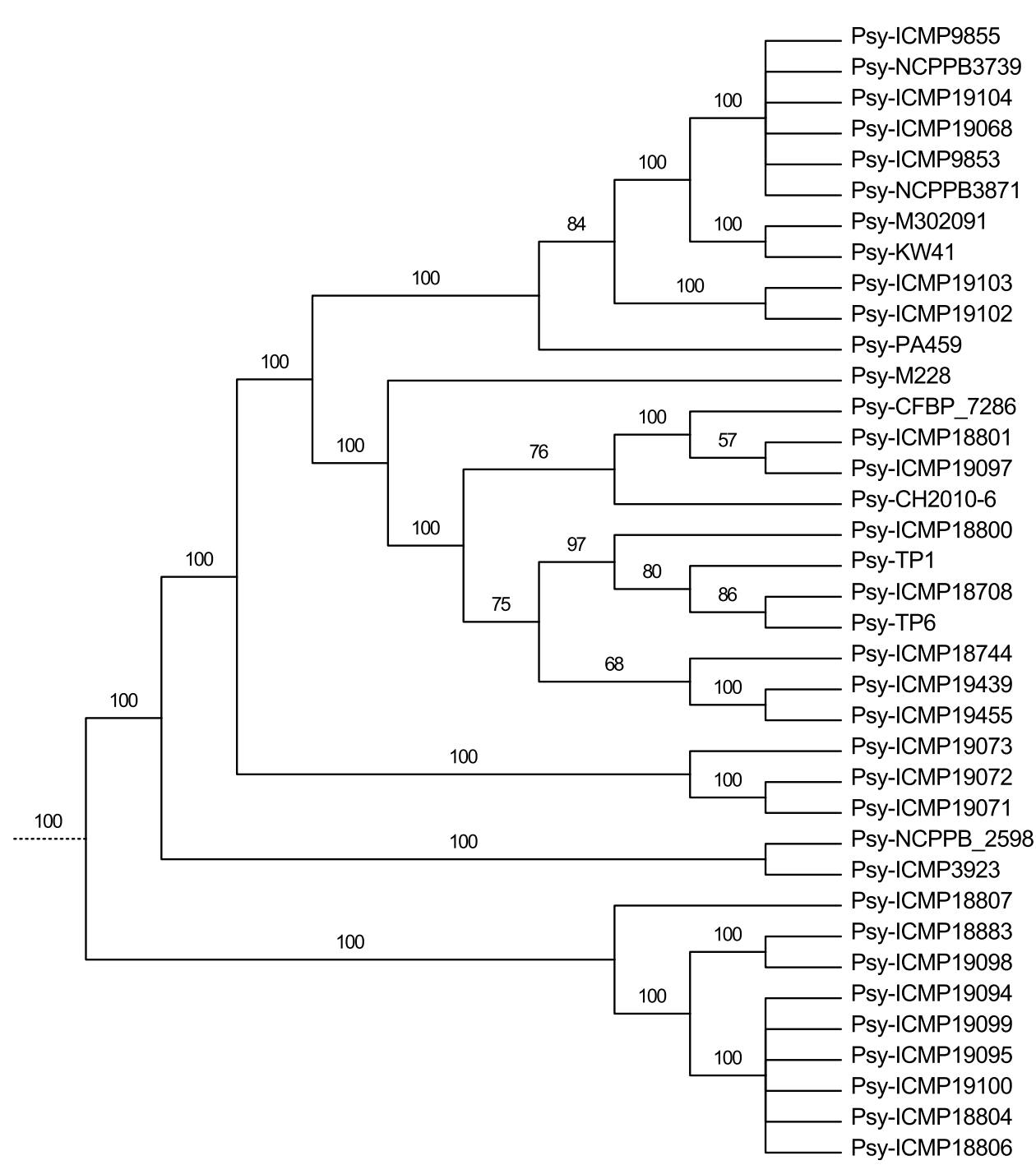

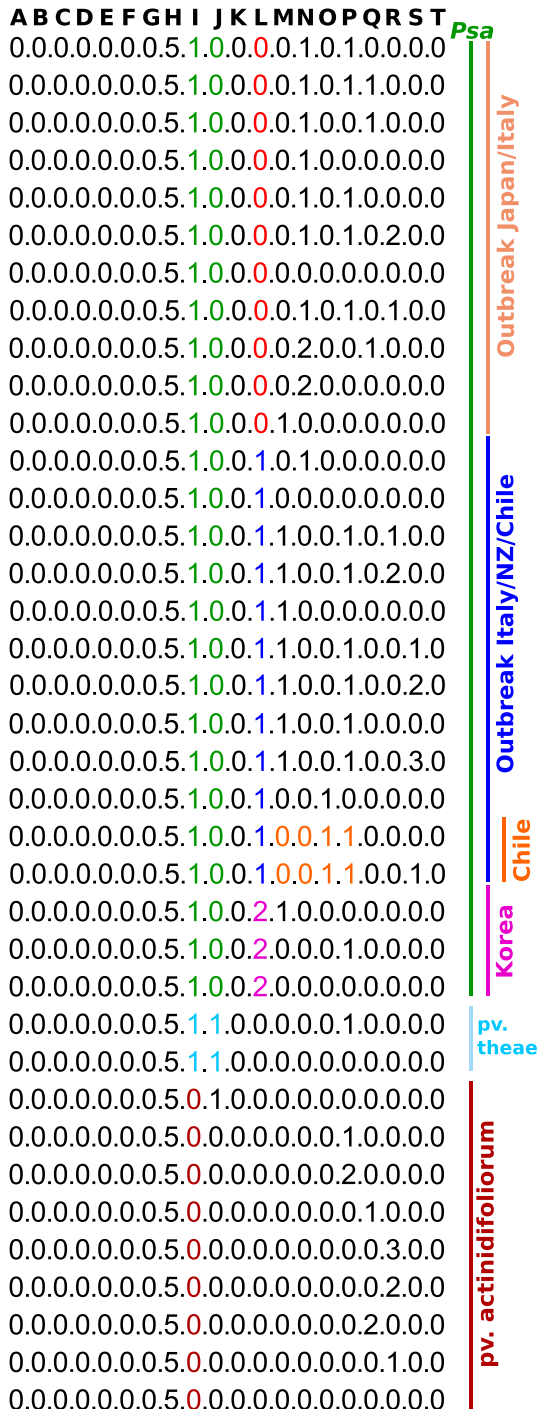

Fig. 7. Section of the Pseudomonas syringae sensu lato core genome tree, life identification numbers (LINs), and current classification of strains including geographic information. LIN positions are labeled at the top of the figure. 
these strains would still not share any LIN position that would reveal that all three belong to clade I. However, note that the ANI between Psy-CC1513 and Psy-CC1466 (85.52\%) is much lower than the ANI currently used to assign strains to the same species (95\%). Therefore, the fact that LINs do not group Psy-CC1513 together with Psy-CC1466, and separate them from Pav-CRAFRUec1, actually correctly reflects that these three strains are equally different from each other. Importantly though, as pointed out in the results section, even when there is not a LIN position that uniquely identifies strains as clade members, membership can still be described by the LINs that correspond to the three subclades.

In a minority of cases though ( 8 of 76), a single LIN position was not sufficient to describe a clade. In these cases, LINs grouped strains slightly differently compared with core genome phylogeny. The reason is that LINs are assigned based on overall genome similarity. Therefore, the most ancestral strain in a clade is sometimes more similar to a strain located immediately outside of the clade than to the other members of the same clade. Such strain thus shares a LIN with the strain outside of the clade and the clade cannot be named using that LIN position. However, as we have shown, even in these cases, LINs could be used to describe a clade by using more than one LIN position. Therefore, all clades in the $P$. syringae tree in Figure 3 could be precisely named using LINs.

This means that if any core genome clade represented any group of bacteria that shared a common trait, for example being pathogenic on a certain host or simply being isolated during the same disease epidemic, this group could be precisely named using LINs.

Our next question was whether the information that is encoded in the assigned LINs is in agreement with the phylogeny inferred from core genomes? As described above, since LINs are assigned based on overall genome similarity and clades in phylogenetic trees are sometimes based on a very small number of informative positions, in some cases, strains shared a LIN with strains on a more basal branch (because they were overall more similar to the strain on the more basal branch) but not with the more derived stains of the clade to which they belonged (because these strains were overall less similar). These differences between phylogeny and LINs are inherent to sequential LIN assignment based on genome identity. We are of the opinion that these small differences between phylogeny and LINs are acceptable in light of the other advantages of LINs, first of all the stability of LINs (i.e., LINs of individual strains do not change upon discovery of new diversity). However, we need to caution that only a relatively small number of genomes was used here. Once LINs are assigned to thousands of closely related $P$. syringae genomes, differences may add up.

The third question, if phylogeny inferred from whole genomes is even representative of evolutionary relationships, may be the most controversial question and almost impossible to answer at this point. It is true that in some groups of bacteria (for example within the genus Streptococcus), wide-spread horizontal gene transfer due to homologous recombination does not allow inference of phylogeny from core genomes without first eliminating recombining regions (Croucher et al. 2011). However, for members of many bacterial genera, core genome trees with clades with high bootstrap support have been built, for example (Epstein et al. 2012; Kumar et al. 2015), including, in fact, the four Pseudomonas core genome trees built as part of the work described here. We think that this is because the sheer number of core genome genes that are inherited vertically "washes out the noise" from recombination events, because individual recombination events generally affect a relatively small number of genes, in different genomic regions, in different subsets of strains, with horizontal transfer occurring in different directions. Also, it is important to keep in mind that core genome trees are based on the alignment of hundreds or thousands of genes compared with single-gene $16 \mathrm{~S}$ trees or MLST trees, based on perhaps seven genes, or rMLST trees, based on 53 genes. Therefore, we believe that core genome trees are for now the best possible representation of evolutionary relationships among bacteria and serve us well when determining how well LINs represent phylogeny. However, we acknowledge that more research needs to be done. Today, this question is being answered differently by different experts; see the opinion paper by Klenk and Göker (2010).

Finally, after finding that LINs reflect phylogenetic relationships (at least those obtained from core genome trees) and showing that, after further optimization, the number of core genome clades reflected in LINs can be further increased, could LINs be used as basis for a new taxonomy that describes and names phylogenetic groups avoiding the concept of species all together? We think so. The examples of $P$. syringae sensu lato and $P$. syringae pv. actinidiae show that LINs can be clearly predictive of phylogeny, of biology, and of the association with specific outbreaks and epidemics. Of course, before permanent LINs can be assigned, many more such comparisons between LINs and biologically and epidemiologically significant groups need to be performed in order to optimize the number of LIN positions and their corresponding thresholds so that the same LIN positions and the same thresholds can be used for a single system for all bacteria in order to avoid confusion. Moreover, it needs to be determined how LINs reflect phylogeny in groups of highly recombining bacteria and in groups of bacteria that either contain members with large plasmids or members with reduced genomes.

Also, while ANI has proven here, and in our previous work (Marakeby et al. 2014), to provide measures of genome similarity that can be used in the assignment of LINs that are predictive of phylogenetic relationships, other measures of genome similarity (or even other measures of relatedness) should be investigated as the basis for LIN assignment. For example, a combination of ANI and aligned genome fractions (as was recently proposed for species delineations [Varghese et al. 2015]) could be used to further improve representation of evolutionary and biologically significant groups by LINs.

The next step we envision for the development of LINs is to create a LIN database to which users can submit genome sequences and metadata. The database would assign LINs to every submitted genome in the order in which genomes are received. Database users would then be able to use the database to associate descriptions (be it a shared phenotype, such as ability to cause canker on kiwifruit plants, or an association with a disease outbreak, such as the current canker of kiwifruit epidemic) with any group of genomes and name that group using the LIN positions that the members of that group share. These LIN-based taxa, or LIN groups, would make it possible to clearly communicate about emerging pathogens (or any other newly discovered group of bacteria) as soon as their genomes become available. Of course, species names could be associated with LIN groups when a LIN group satisfies the criteria for a named species. Therefore, LIN groups would not in any way replace current taxonomy but actually facilitate species descriptions and complement current taxonomy. LIN groups would simply make it much easier and faster to describe and name any group of bacteria, in particular, plant pathogenic bacteria, without having to wait until a peer-reviewed named species is described.

Importantly, while it would be possible to define new LIN groups in the database at any time and it would be possible to change associations of phenotypes with LIN groups any time, the LINs assigned to the individual genomes would always remain the same providing a stable genome-similarity based framework to which all past, current, and future LIN groups would be linked. This would eliminate one of the main problems plant pathologists have with the current taxonomic system: the confusion when a well-known pathogen strain with a familiar species name is assigned to a new species with a new name because of a new or revised species description (Baltrus 2016). 


\section{LITERATURE CITED}

Altschul, S. F., Madden, T. L., Schaffer, A. A., Zhang, J., Zhang, Z., Miller, W., and Lipman, D. J. 1997. Gapped BLAST and PSI-BLAST: A new generation of protein database search programs. Nucleic Acids Res. 25:3389-3402.

Baltrus, D. A. 2016. Divorcing strain classification from species names. Trends Microbiol. 24:431-439.

Berge, O., Monteil, C. L., Bartoli, C., Chandeysson, C., Guilbaud, C., Sands, D. C., and Morris, C. E. 2014. A user's guide to a data base of the diversity of Pseudomonas syringae and its application to classifying strains in this phylogenetic complex. PLoS One 9:e105547.

Bull, C. T., Clarke, C. R., Cai, R., Vinatzer, B. A., Jardini, T. M., and Koike, S. T. 2011. Multilocus Sequence Typing of Pseudomonas syringae sensu lato confirms previously described genomospecies and permits rapid identification of $P$. syringae pv. coriandricola and $P$. syringae pv. apii causing bacterial leaf spot on parsley. Phytopathology 101:847-858.

Bull, C. T., Manceau, C., Lydon, J., Kong, H., Vinatzer, B. A., and Fischer-Le Saux, M. 2010. Pseudomonas cannabina pv. cannabina pv. nov., and Pseudomonas cannabina pv. alisalensis (Cintas Koike and Bull, 2000) comb. nov., are members of the emended species Pseudomonas cannabina (ex Sutic \& Dowson 1959) Gardan, Shafik, Belouin, Brosch, Grimont \& Grimont 1999. Syst. Appl. Microbiol. 33:105-115.

Butler, M. I., Stockwell, P. A., Black, M. A., Day, R. C., Lamont, I. L., and Poulter, R. T. M. 2013. Pseudomonas syringae pv. actinidiae from recent outbreaks of kiwifruit bacterial canker belong to different clones that originated in China. PLoS One 8:e57464.

Chapman, J., Taylor, R., and Alexander, B. 2011. Second report on characterization of Pseudomonas syringae pv. actinidiae (Psa) isolates in New Zealand. Ministry of Agriculture and Forestry Report, March 2011.

Chapman, J. R., Taylor, R. K., Weir, B. S., Romberg, M. K., Vanneste, J. L., Luck, J., and Alexander, B. J. R. 2012. Phylogenetic relationships among global populations of Pseudomonas syringae pv. actinidiae. Phytopathology 102:1034-1044.

Cintas, N. A., Koike, S. T., and Bull, C. T. 2002. A new pathovar, Pseudomonas syringae pv. alisalensis pv. nov., proposed for the causal agent of bacterial blight of broccoli and broccoli raab. Plant Dis. 86:992-998.

Croucher, N. J., Harris, S. R., Fraser, C., Quail, M. A., Burton, J., van der Linden, M., McGee, L., von Gottberg, A., Song, J. H., Ko, K. S., Pichon, B., Baker, S., Parry, C. M., Lambertsen, L. M., Shahinas, D., Pillai, D. R., Mitchell, T. J., Dougan, G., Tomasz, A., Klugman, K. P., Parkhill, J., Hanage, W. P., and Bentley, S. D. 2011. Rapid pneumococcal evolution in response to clinical interventions. Science 331:430-434.

Cunty, A., Poliakoff, F., Rivoal, C., Cesbron, S., Fischer-Le Saux, M., Lemaire, C., Jacques, M. A., Manceau, C., and Vanneste, J. L. 2014. Characterisation of Pseudomonas syringae pv. actinidiae (Psa) isolated from France and assignment of strains Psa biovar 4 to a de novo pathovar: Pseudomonas syringae pv. actinidifoliorum pv. nov. Plant Pathol. 64:582-596.

Epstein, B., Branca, A., Mudge, J., Bharti, A. K., Briskine, R., Farmer, A. D., Sugawara, M., Young, N. D., Sadowsky, M. J., and Tiffin, P. 2012. Population genomics of the facultatively mutualistic bacteria Sinorhizobium meliloti and S. medicae. PLoS Genet. 8:e1002868.

Gardan, L., Shafik, H., Belouin, S., Broch, R., Grimont, F., and Grimont, P. A. 1999. DNA relatedness among the pathovars of Pseudomonas syringae and description of Pseudomonas tremae sp. nov. and Pseudomonas cannabina sp. nov. (ex Sutic and Dowson 1959). Int. J. Syst. Bacteriol. 49:469-478.

Goris, J., Konstantinidis, K. T., Klappenbach, J. A., Coenye, T., Vandamme, P., and Tiedje, J. M. 2007. DNA-DNA hybridization values and their relationship to whole-genome sequence similarities. Int. J. Syst. Evol. Microbiol. 57:81-91.

Hodcroft, E. 2015. TreeCollapserCL 4. Available online. emmahodcroft.com/ TreeCollapseCL.html

Huson, D. H., and Bryant, D. 2006. Application of phylogenetic networks in evolutionary studies. Mol. Biol. Evol. 23:254-267.

Jolley, K., and Maiden, M. 2010. BIGSdb: Scalable analysis of bacterial genome variation at the population level. BMC Bioinformatics 11:595.

Jolley, K. A., Bliss, C. M., Bennett, J. S., Bratcher, H. B., Brehony, C., Colles, F. M., Wimalarathna, H., Harrison, O. B., Sheppard, S. K., Cody, A. J., and Maiden, M. C. J. 2012. Ribosomal multilocus sequence typing: Universal characterization of bacteria from domain to strain. Microbiology 158: 1005-1015.

Katoh, K., and Standley, D. M. 2013. MAFFT multiple sequence alignment software version 7: Improvements in performance and usability. Mol. Biol. Evol. 30:772-780.

Klenk, H. P., and Göker, M. 2010. En route to a genome-based classification of archaea and bacteria? Syst. Appl. Microbiol. 33:175-182.
Konstantinidis, K. T., and Tiedje, J. M. 2005. Genomic insights that advance the species definition for prokaryotes. Proc. Natl. Acad. Sci. USA 102: 2567-2572.

Kumar, N., Lad, G., Giuntini, E., Kaye, M. E., Udomwong, P., Shamsani, N. J., Young, J. P. W., and Bailly, X. 2015. Bacterial genospecies that are not ecologically coherent: Population genomics of Rhizobium leguminosarum. Open Biol. 5:140133.

Maiden, M. C., Bygraves, J. A., Feil, E., Morelli, G., Russell, J. E., Urwin, R., Zhang, Q., Zhou, J., Zurth, K., Caugant, D. A., Feavers, I. M., Achtman, M., and Spratt, B. G. 1998. Multilocus sequence typing: A portable approach to the identification of clones within populations of pathogenic microorganisms. Proc. Natl. Acad. Sci. USA 95:3140-3145.

Marakeby, H., Badr, E., Torkey, H., Song, Y., Leman, S., Monteil, C. L., Heath, L. S., and Vinatzer, B. A. 2014. A system to automatically classify and name any individual genome-sequenced organism independently of current biological classification and nomenclature. PLoS One 9:e89142.

Marcelletti, S., Ferrante, P., Petriccione, M., Firrao, G., and Scortichini, M. 2011. Pseudomonas syringae pv. actinidiae draft genomes comparison reveal strain-specific features involved in adaptation and virulence to Actinidia species. PLoS One 6:e27297.

Mayden, R. L. 1997. A hierarchy of species concepts: The denoue- ment in the saga of the species problem. Pages 324-381 in: Species: The Units of Biodiversity. M. F. Claridge, H. A. Dawah, and M. R. Wilson, eds. Chapman and Hall, London.

Mayr, E. 1942. Systematics and the Origin of Species. Columbia University Press, New York.

Mazzaglia, A., Renzi, M., and Balestra, G. M. 2011. Comparison and utilization of different PCR-based approaches for molecular typing of Pseudomonas syringae pv. actinidiae strains from Italy. Can. J. Plant Pathol. 33:8-18.

Mazzaglia, A., Studholme, D. J., Taratufolo, M. C., Cai, R., Almeida, N. F., Goodman, T., Guttman, D. S., Vinatzer, B. A., and Balestra, G. M. 2012. Pseudomonas syringae pv. actinidiae (PSA) isolates from recent bacterial canker of kiwifruit outbreaks belong to the same genetic lineage. PLoS One 7:e36518.

McCann, H. C., Rikkerink, E. H. A., Bertels, F., Fiers, M., Lu, A., Rees-George, J., Andersen, M. T., Gleave, A. P., Haubold, B., Wohlers, M. W., Guttman, D. S., Wang, P. W., Straub, C., Vanneste, J., Rainey, P. B., and Templeton, M. D. 2013. Genomic analysis of the kiwifruit pathogen Pseudomonas syringae pv. actinidiae provides insight into the origins of an emergent plant disease. PLoS Pathog. 9:e1003503.

Richter, M., and Rosselló-Móra, R. 2009. Shifting the genomic gold standard for the prokaryotic species definition. Proc. Natl. Acad. Sci. 106: 19126-19131.

Rossello-Mora, R., and Amann, R. 2001. The species concept for prokaryotes. FEMS Microbiol. Rev. 25:39-67.

Sarkar, S. F., and Guttman, D. S. 2004. Evolution of the core genome of Pseudomonas syringae, a highly clonal, endemic plant pathogen. Appl. Environ. Microbiol. 70:1999-2012.

Scortichini, M. 1994. Occurrence of Pseudomonas syringae pv. actinidiae on kiwifruit in Italy. Plant Pathol. 43:1035-1038.

Stamatakis, A. 2014. RAxML version 8: A tool for phylogenetic analysis and post-analysis of large phylogenies. Bioinformatics 30:1312-1313.

Takikawa, Y., Serizawa, S., and Ichikawa, T. 1989. Pseudomonas syringae pv. actinidiae pv. nov.: The causal bacterium of canker of kiwifruit in Japan. Ann. Phytopathol. Soc. Jpn. 55:437-444.

Thompson, C., Amaral, G., Campeão, M., Edwards, R., Polz, M., Dutilh, B., Ussery, D., Sawabe, T., Swings, J., and Thompson, F. 2015. Microbial taxonomy in the post-genomic era: Rebuilding from scratch? Arch. Microbiol. 197:359-370.

Varghese, N. J., Mukherjee, S., Ivanova, N., Konstantinidis, K. T., Mavrommatis, K., Kyrpides, N. C., and Pati, A. 2015. Microbial species delineation using whole genome sequences. Nucleic Acids Res. 43:6761-6771.

Vinatzer, B. A., and Bull, C. T. 2009. The impact of genomics approaches on our understanding of diversity and taxonomy of plant pathogenic bacteria. Pages 37-61 in: Plant Pathogenic Bacteria: Genomics and Molecular Biology. R. W. Jackson, ed. Horizon Scientific Press, Norfolk, UK.

Vinatzer, B. A., Monteil, C. L., and Clarke, C. R. 2014. Harnessing population genomics to understand how bacterial pathogens emerge, adapt to crop hosts, and disseminate. Annu. Rev. Phytopathol. 52:19-43.

Weisberg, A. J., Marakeby, H., Heath, L. S., and Vinatzer, B. A. 2015. Similarity-based codes sequentially assigned to ebolavirus genomes are informative of species membership, associated outbreaks, and transmission chains. Open Forum Infect. Dis. 2(1). doi:10.1093/ofid/ofv024

Young, J. M., Dye, D. W., Bradbury, J. F., Panagopoulos, C. G., and Robbs, C. F. 1978. A proposed nomenclature and classification for plant pathogenic bacteria. N.Z. J. Agric. Res. 21:153-177. 